\title{
\#ALL versus ALL in American Sign Language (ASL)
}

\author{
Margaret Ruth Crabtree \& Ronnie B. Wilbur*
}

\begin{abstract}
This paper extends a visible pattern ('iconicity') that has been observed in sign language verbs and adjectives to quantification in American Sign Language (ASL). The Event Visibility Hypothesis (EVH) states that boundedness is morphophonologically encoded in articulation of a rapid deceleration of movement at the end of a sign (aka end-marking). Here the EVH is applied to the two ASL quantifiers glossed \#ALL and ALL. Doing so accounts for the semantic distinction between them: ALL is definite (bounded), whereas \#ALL is underspecified for definiteness (unbounded).
\end{abstract}

Keywords. sign language; ASL; American Sign Language; quantification; universal quantification; ALL; Event Visibility Hypothesis; EVH; iconicity

1. Introduction. The Event Visibility Hypothesis (EVH) (Wilbur 2008) states that boundedness is morphophonologically encoded in articulation of a rapid deceleration of movement at the end of a sign (aka end-marking). End-marking is observed in signs with contact between hand and a body part, abrupt change in movement, setting/location change, aperture or orientation change in telic verbs (Malaia \& Wilbur 2012; Malaia, Wilbur \& Milković 2013), adjectives in the excessive construction (e.g., 'too far to walk') (Wilbur, Malaia \& Shay 2012), count nouns (Kuhn, Geraci, Schlenker \& Strickland 2018), and tentatively spoken English concrete nouns ending in stops (Kuhn et al. 2018). The EVH is the only type of iconicity described for sign language that is accessible without cultural and/or conversational context. The iconicity is so robust that it is accessible to non-signers (Strickland, Geraci, Chemla, Schlenker, Kelepir \& Pfau 2015). We extend the observed domain of the EVH to another part of speech: quantifiers.

The EVH model provides a novel semantic distinction for \#ALL and ALL, signs which are generally considered synonymous (e.g. Liskova 2017:104). The citation articulation of \#ALL lacks boundedness (Figure 1), in contrast to ALL, which is signed with the boundedness feature: sign-final hand contact (Figure 2). The effect of boundedness on these quantifiers highly (but not $100 \%$ ) correlates with the indefinite (-def; \#ALL) versus definite (+def; ALL) distinction of English.

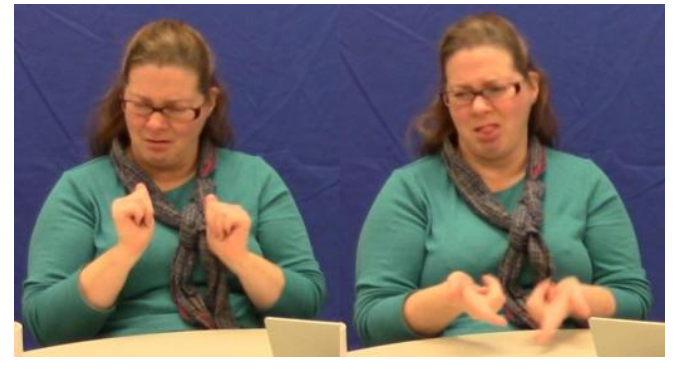

Figure 1. \#ALL

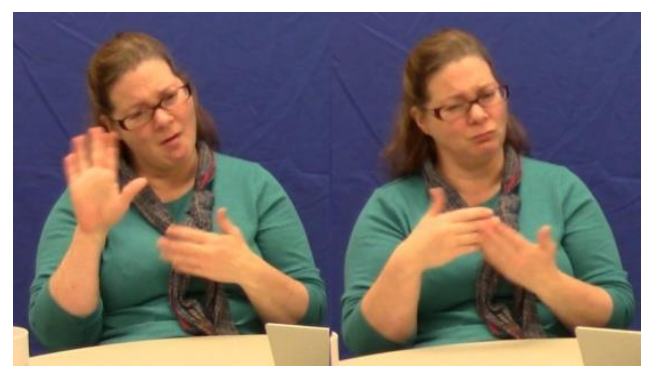

Figure 2. ALL

\footnotetext{
* Authors: Margaret Ruth Crabtree, Purdue University (crabtrm@purdue.edu) \& Ronnie B. Wilbur, Purdue University (wilbur@purdue.edu).
} 
In Section 2, we provide background on the signs \#ALL and ALL. Section 3 provides an introduction to telicity. Section 4 contains our data and analysis. Section 5 is discussion and Section 6 concludes.

2. Background on \#ALL and ALL. Generally speaking, \#ALL and ALL are said to be distinguished only phonologically. \#ALL is derived from fingerspelling, can be signed either one or two handed, and can freely colocate in space to provide additional information about the size, location, and configuration of the nominal being modified. One might be tempted to categorize the change of articulation from the handshape of ' $A$ ' to that of ' $L$ ' as 'change of aperture' (an end-marking option), but this handshape change occurs sign initially, rather than sign finally, thus it cannot correlate temporally with rapid deceleration marking the end of the sign. In contrast, ALL is a native two-handed sign with contact at the end, made in front of the body. It is this relatively sudden stop of the dominant hand (DH) upon contact with the non-dominant hand (NDH), that contains the rapid deceleration resulting in end-marking. ALL is generally unable to colocate, although Liskova (2017:169) reports that it may be signed slightly to the horizontal left and right to agree with two different contrasted arguments.

As for previous analysis, Abner \& Wilbur (2017) claim that three universal quantifiers of ASL are valued for the feature [distributivity]. They claim that \#ALL (+/- distributive) and $\mathrm{EACH}$ (+/- distributive) are compatible with distributive interpretations, but ALL (-distributive) (which they gloss as ALL-CIRCLE) can only be used with a collective reading. (1) is an example of this (their example).

(1) (Wilbur \& Abner 2017:43)

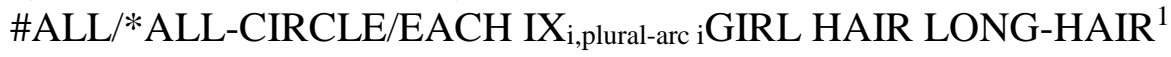

'All the girls have long hair.'

However Liskova (2017) reports that ALL (ALL-CIRCLE), like \#ALL, is acceptable with a distributive interpretation. Her examples of this are provided here as $(2 a)$ and $(2 b)$.

(2) (Liskova 2017:167)

Stimulus sentence: All of the dogs are sleeping.

\section{a. ALL \#DOGS SLEEP \\ $\mathrm{t}$ \\ b. DOG IX[pl] ALL SLEEP}

After finding this exception to the model provided by Abner \& Wilbur (2017), Liskova (2017) goes into great detail about the numerous phonetic variations of \#ALL that she observes, but eventually she does conclude that \#ALL and ALL are semantically synonymous.

The stated aim of Liskova (2017:57) is to test ASL quantifiers for sensitivity to the following features: count/mass, concrete/abstract, and animacy. In her methods Liskova (2017:58) clearly states that all of her English elicitation stimuli contain only definite determiner phrases (DPs), all with the determiner 'the'. Thus she would have collected no generic or habitual statements, those being the ones that would have explicitly required the \#ALL universal

\footnotetext{
${ }^{1}$ The ASL here is glossed in the typical format of written sign language data: signs are written in all caps; dashed words indicate that a single sign requires multiple English words for translation; '(fs)_' precedes a word to indicate a fingerspelled English word; '\#' precedes a word to indicate a fingerspelled English word that has been lexicalized into ASL; IX stands for index which is deictic pointing. The indices in our data are much more narrowly glossed than is generally considered necessary. They are marked for number, plurality, definiteness, and specificity.
} 
quantifier, as we will show. Nonetheless, her data do in fact show, as we discuss in Section 4, that both \#ALL and ALL may be used in most definite DPs (the only DPs she collected).

This background information on universal quantification in ASL only touches on the underexplored relationships between \#ALL and ALL and the elements of nominal features. We are claiming that ASL quantifiers share articulatory and semantic features with verbs, although they are not deverbal in any known way. The basis of these shared features can be found in telicity. In the following section we introduce telicity and how this feature classically tied to verbs has also always been tied to quantifiers, strengthening the theoretical basis of our claim.

3. Introduction to Telicity. Telicity refers to whether the event described in a sentence has a natural end state or not. The basic possibilities are: telic and atelic. A telic event ( 3 and 4 ) is one that has a natural end point and is possible to either partially or $100 \%$ complete. An atelic event (5 and 6) has no natural point of completion.

(3) Mary died. (telic)

(4) Mary ate two bananas. (telic)

(5) Mary slept. (atelic)

(6) Mary built houses. (atelic)

All languages distinguish these two types of events, but the syntax, phonology, and morphology used to do so varies wildly from language to language. Thus to be sure of the telicity, language-specific syntactic tests must be performed. The simplest test is to check whether the structure describing the event is compatible with 'for X amount of time' or 'in X amount of time', although other tests may be needed as well. In (7), 'sleeping' doesn't naturally complete at any predetermined point, so it is more natural to describe an event of sleeping as occurring 'for five minutes' (7a). In contrast, in (8b) 'to eat two bananas' is naturally completed when both the bananas are eaten up (whether or not that ever occurs) so (8b) is more natural.

(7) a. Mary slept for five minutes.

b. *Mary slept in five minutes.

(8) a. *Mary ate two bananas for five minutes.

b. Mary ate two bananas in five minutes.

In the cases of (3) and (5) the telicity comes entirely from the verb, this is called 'lexical aspect' or 'Aktionsart'. In the cases of (4) and (6) the telicity of the event is constructed by including additional information, in this case the object argument. In English 'eat' can be part of either a telic or atelic event, (8b) and (9a) respectively.

(9) a. Mary ate bananas for five minutes.

b. *Mary ate bananas in five minutes.

In the telic example with 'eat', (8), it is the quantifier that has given a natural end point to the event. The natural end point to 'eating two bananas' is when two bananas are eaten, and not any other amount. Quantifiers are thus a core part of telicity as they can bound events and create telic end points. It is naturally expected for this relationship to be maintained in sign languages. What is fascinating here is that the shared feature of providing telicity can be more generally described as boundedness, and that this feature realizes via the same morphophonology in both verbs and quantifiers in ASL. 
4. Data and Analysis. Our claim is that a distinction very similar to definiteness in English is being made by signers when they choose between the signs \#ALL and ALL. Explicitly, we call this feature 'boundedness'. We introduced Liskova (2017)'s dataset that was obtained without any indefinite stimuli and thus contained no distinctions between these two signs. Naturalistic ASL data is also not suited to search for this distinction as there is no way to judge what definiteness the signer intended to convey.

4.1. DATA. Our analysis began with corpus data we had collected in 2015, on the topic of 'quantifiers, generics, and habituals'. This set was constructed based on asking our informant to interpret English stimuli which conveyed fine distinctions in definiteness, quantification, and aspect. Thus we could infer that the intended definiteness of the ASL utterances matched that of their English stimuli.

In order to confirm these inferences and understand what kind of context each use of 'all' implied, we re-interviewed the same informant in 2019. In this interview we offered various orderings of written ASL gloss sentences composed of '(fs)_MARY, COOKIE, \#ALL/ALL, EAT, (FINISH)'. We requested these to be signed and then judged for grammaticality, and then any required contexts for cases of borderline grammaticality to be described. For these interview data, acceptable sentences are not marked; ungrammatically is marked with '*'; and ASL sentences that were judged ungrammatical, but then a context was offered in which the sentence would be acceptable, are marked with '?'.

Thus some of the below ASL data are based on written English stimuli and some are based on written ASL gloss stimuli, each example is labeled accordingly. Some stimuli are rated ungrammatical as-is, but the signer offers alternatives occasionally, so the grammaticality ratings of a stimulus and its interpretation do not always match. For additional clarity, translations are given for the ASL that was actually signed.

4.2. ANALYSIS OF ALL. The following data support the claim that the universal quantifier ALL has the feature 'boundedness', and that it requires at least one additional bounded element to license its presence in a structure. This additional element may be one of two things: an aspect marker that end-marks an event or an index which is both definite ${ }^{2}$ and specific ${ }^{3}$. A typical example of ALL appearing within an appropriate context can be seen in (10).

(10) Stimulus (written ASL gloss): (fs)_MARY ALL COOKIE FINISH EAT

ASL: (fs)_MARY ALL COOKIE FINISH EAT

Context: 'Mary ate everything, nothing's left'.

Translation: 'Mary finished eating all the cookies.'

\footnotetext{
${ }^{2}$ There is still much debate as to whether ASL is a language that conveys definiteness or not; our claims clearly intersect this debate as our analysis requires that ASL does convey definiteness. Aside from this theoretical stance, we will not explore this debate further here.

${ }^{3}$ The data from the 2015 and 2019 corpora have their indices (IX) marked mainly following MacLaughlin (1997) for definiteness (downward pointing) and specificity (statically pointing to a location in space). However, MacLaughlin (1997:122) does not distinguish 'plural specific' and 'plural nonspecific' indices. We do make this distinction. We use her model to do so by slightly expanding on her definition of pointing to a location that allows circular plural indices to be defined as specific and arc (incomplete circle) plural indices to be defined as nonspecific. Specific index: the beginning and ending of the index sign share the same location, viz. static point or complete circle. The features 'definite' and 'specific' together correlate with the semantic feature we call 'boundedness'.
} 
In (10) the main verb is 'eat' and what is glossed as FINISH is a grammaticalized terminative aspect marker, from the lexical verb FINISH. Here FINISH licenses ALL to appear in the construction. A polar question variant of (10), with significantly different word order can be seen in (11).

(11) Stimulus (written English): 'Had Sally eaten all the cookies?'

ASL: (SALLY)-IX-i EAT ALL COOKIE FINISH IX-i 3.SG.DEF.SPEC

3.SG.DEF.SPEC

Translation: 'Did she finish eating all the cookies?'

The absence of FINISH leads to ungrammaticality, as can be seen in (12).

(12) Stimulus (written ASL gloss): * (fs)_MARY ALL COOKIE EAT

ASL: *(fs)_MARY ALL COOKIE EAT

FINISH is not the only aspect marker which can be used. After stating that (12) was ungrammatical, the informant offered a slight modification based on the stimulus in (12), given in (13). Here she gives us an example of another aspect marker that licenses the appearance of ALL, BECOME-EMPTY. This sign indicates the change of state to emptiness/nothingness. Like FINISH, it can also otherwise appear as a main predicate.

\section{(13) Stimulus (written ASL gloss): * (fs)_MARY ALL COOKIE EAT}

ASL: (fs)_MARY ALL COOKIE EAT BECOME-EMPTY

Translation: 'Mary ate up all the cookies.'

Besides the aspect signs, our data show that boundedness can also be provided by definite specific indexes that colocate with the quantified nominal. The English stimuli in (14) and (15) form a very near minimal pair, the former had 'all of the workshops' in contrast to 'all workshops' in the latter. Both could be signed with \#ALL but only (14) could be signed with ALL. The ASL in (14) and (15) differ mainly by the (bold) definite specific index in (14) which is lacking from (15).

(14) Stimulus (written English): 'Teachers must attend all of the workshops.'
ASL: WORKSHOP-i LIST-i IX-i HAVE IX-i 3.SG.DEF.SPEC 3.PL ${ }^{4}$.DEF.NSPEC 3 3.PL 5 .DEF.SPEC
REQUIRE GO \#ALL/ALL

Translation: 'As for this whole list of workshops, all the teachers are required to go to all of them.'

(15) Stimulus (written English): 'Teachers must attend all workshops.'

ASL: WORKSHOP-i IX-i LIST-i IX-i TEACHER REQUIRE GO \#ALL-i

3.PL ${ }^{6}$.DEF.NSPEC 3.PL 7 .DEF.NSPEC

Translation: 'As for the list of workshops, teachers are required to go to all of them.'

Both (14) and (15) have an index that moves downward, tracing the list of workshops which we judge to be definite and nonspecific. It is only in (14) that there is a definite specific index.

\footnotetext{
4 The plural index starts pointed at the non-dominant FlatB hand and then moves vertically downwards.

5 The plural index points downward and traces a circle.

6 The plural index points downward and moves along a horizontal number line.

${ }^{7}$ The plural index points downward and moves vertically downwards.
} 
A parallel pair of example ASL sentences may be seen in (16) and (17). The informant provided these two in response to a single English stimulus: one interpretation each for ALL and \#ALL. As with the previous example pair, it is only the sentence with ALL that contains a (bold) definite specific index.

(16) Stimulus (written English): 'All the people in the room were drunk.'

ASL: PEOPLE-i IX-i ROOM-i ALL DRUNK

PL 3.DEF.SPEC

Translation: 'As for the people in the room, all were drunk.'

(17) Stimulus (written English): 'All the people in the room were drunk.'

ASL: ROOM PARTY PEOPLE-i LARGE-GROUP-i \#ALL-i DRUNK

PL

Translation: 'In the room there is a party, there is a large group of people all drunk.'

In (14) and (16), the 'ALL with index' examples, the index was articulated as a static downward point. This can be interpreted as singular or plural based on the context. However the next example, (18), has an explicitly plural definite specific index which colocates with the quantified nominal. This index is articulated as a complete circle, not to be confused with a linear line or an arc segment covering significantly less than 360 degrees. The stop at the origin point in fact appears rapid, and likely qualifies as end-marking via rapid deceleration, but further motion capture data collection would be required to confirm this.

(18) Stimulus (written English): 'Each teacher must attend all workshops.'

ASL: ALL WORKSHOP-i GROUP-i IX-i EACH-j TEACHER-j IX-j GO++ ALL MUST 3.PL ${ }^{8}$.DEF.SPEC 3.PL ${ }^{9}$.DEF.SPEC

Translation: 'All of these workshops, each teacher must go to all of them.'

(18) is further evidence that it is specific shared features, in this case definite and specific, that convey boundedness, and these features do not prohibit other non-conflicting (in this case plurality) features from co-occurring. For thoroughness' sake, we also have an example of a plural definite non-specific index that was judged ungrammatical with ALL, (19), which is another response to the same stimulus used in examples (12) and (13).

(19) Stimulus (written ASL gloss): * (fs)_MARY ALL COOKIE EAT

ASL: * (fs)_MARY EAT ALL COOKIE-i IX-i

3.PL ${ }^{10}$.DEF.NSPEC

We have analyzed ASL data containing ALL and attempted to find the common features shared in the grammatical utterances which were lacking in the ungrammatical ones. We observed that two types of signs co-occurred with ALL in grammatical cases, and propose that what unites these two are boundedness, which we argue is necessary to license ALL. The specific signs we observe that carry the feature boundedness are end-marking aspect markers (viz. FINISH and BECOME-EMPTY) and definite specific indices (both plural and underspecified for singular/plural).

\footnotetext{
8 The plural index points downward and traces a circle.

${ }^{9}$ The plural index points downward and traces a circle.

${ }^{10}$ The plural index points downward and moves along a horizontal number line.
} 
4.3. ANALYSIS OF\#ALL. Based on the data we have looked at, in contrast to ALL's specific licensing requirements, \#ALL can appear in nearly any context. It was only ungrammatical as a modifier of the most bounded nominals, that is, \#ALL was unable to modify a fully affected argument. We claim that the lack of end-marking in the articulation of \#ALL is what prevents this sign from being an all-purpose flexible universal quantifier.

\#ALL has the added flexibility, in contrast to ALL, of colocation (changing place of articulation to match a co-indexed nominal), of one or two-handed signing (allowing smaller and larger sets to be indicated), as well as the ability to use vertical space to indicate abstractly larger set sizes (Davidson \& Gagne 2014). These are the known factors that influence which sign is chosen in those cases where either \#ALL or ALL would be grammatical.

The lack of end-marking/boundedness makes \#ALL the only choice in cases like (20), if you want an unbounded universal statement. ${ }^{11}$

(20) Stimulus (written English): 'All cheese contains protein.'

ASL: CHEESE HAVE (fs)_PROTEIN \#ALL

Translation: 'All cheese has protein.'

Previously as (14) and (15), repeated here as (21) and (22) respectively, the English of the near minimal pair of stimuli can each be translated with \#ALL. For both, the colocation option of \#ALL is put to use, vertically colocating with the sign LIST, which specifies that a series of workshops are required.

(21) Stimulus (written English): 'Teachers must attend all of the workshops.'

ASL: WORKSHOP-i LIST-i IX-i HAVE IX-i IX-j TEACHER-j 3.SG.DEF.SPEC 3.PL ${ }^{12}$.DEF.NSPEC 3. PL $^{13}$.DEF.SPEC

REQUIRE GO \#ALL/ALL

Translation: 'As for this whole list of workshops, all the teachers are required to go to all of them.'

(22) Stimulus (written English): 'Teachers must attend all workshops.'

ASL: WORKSHOP-i IX-i LIST-i IX-i TEACHER REQUIRE GO \#ALL-i

3.PL ${ }^{14}$.DEF.NSPEC $3 . P L^{15}$.DEF.NSPEC

Translation: 'As for the list of workshops, teachers are required to go to all of them.'

The limits of \#ALL are seen in the context sensitive (23) and ungrammatical (24). For (23), the informant explains that in this example 'cookie' receives a 'kind' reading, allowing a universal unbounded interpretation of \#ALL to co-occur with bounded FINISH. In (24) that interpretation is not possible as the combination of PLATE and BECOME-EMPTY explicitly convey completive aspect and denote a completely affected argument. This blocks the 'kind' reading.

\footnotetext{
${ }^{11}$ Following-up with the informant revealed that ALL could be substituted grammatically for \#ALL for the ASL in (20), but it could only refer to 'the cheese that is in front of you', viz. 'a bounded quantity of cheese' as predicted.

12 The plural index starts pointed at the non-dominant FlatB hand and then moves vertically downwards.

${ }^{13}$ The plural index points downward and traces a circle.

${ }^{14}$ The plural index points downward and moves along a horizontal number line.

15 The plural index points downward and moves vertically downwards.
} 
(23) Stimulus (written ASL gloss): ? (fs)_MARY \#ALL COOKIE FINISH EAT

ASL: ? (fs)_MARY \#ALL COOKIE FINISH EAT

Context: 'Mary finishes trying all the types of cookies.'

Translation: 'Mary finished eating (each kind of) cookies.'

(24) Stimulus (written ASL gloss): (fs)_MARY \#ALL COOKIE FINISH EAT ASL: * PLATE (fs)_MARY \#ALL COOKIE FINISH EAT BECOME-EMPTY

4.4. LOOKING BACK AT LISKOVA (2017). In Section 2, we mentioned that Liskova (2017)'s analysis of universal quantifiers in ASL was based on data collected using only definite English stimuli, and that she could not find any syntactic or semantic differences between \#ALL and ALL. Here we analyzed both definite and indefinite stimuli and were able to provide a model that explained the distribution of \#ALL and ALL. ALL could only be used in contexts that licensed it, by means of another bounded element. \#ALL rather seems permissible in nearly any context, save the most bounded when the nominal would be fully affected. Thus, our results do match Liskova (2017)'s observations that both \#ALL and ALL freely appear in response to definite stimuli with a single verbal element, but we were able to go beyond this. We explain the distribution of \#ALL and ALL in complex sentences wherein both signs were not equally permitted.

5. Discussion. \#ALL and ALL are not in complementary distribution, but they also are not equally able to appear in all contexts, as they do convey different values for boundedness. ALL requires additional boundedness from end-marking aspect or a definite specific index, whereas \#ALL is disallowed by completives; it cannot modify a fully affected argument. In all examples \#ALL was associable with an unbounded universal interpretation. The partial overlap of permissible contexts of these non-synonymous signs allows for the minimal pair of ASL sentences in (25) and (26), a single structure where either sign is acceptable but with different interpretations.

(25) Stimulus (written ASL gloss): ? (fs)_MARY \#ALL COOKIE FINISH EAT ASL: ? (fs)_MARY \#ALL COOKIE FINISH EAT

Context: 'Mary finishes trying all the types of cookies.'

Translation: 'Mary finished eating (each kind of) cookie.'

(26) Stimulus (written ASL gloss): (fs)_MARY ALL COOKIE FINISH EAT

ASL: (fs)_MARY ALL COOKIE FINISH EAT

Context: 'Mary ate everything, nothing's left.'

Translation: 'Mary finished eating all the cookies.'

The phenomenon of multiple, difficult to semantically/syntactically distinguish, universal quantifiers is not unique to ASL. Three are reported for Turkish Sign Language (TID) (Saral \& Kelepir 2019, Saral 2019); they also appear to split between colocatable and not. This is in reference to Section 2 where we provided background on \#ALL and ALL. Historically 'colocatable versus not colocatable' had been the main distinction identified in the literature to distinguish \#ALL from ALL in ASL.

6. Conclusion. ASL has two universal quantifiers both commonly glossed as 'all': \#ALL (unbound) and ALL (bound). They obey morphophonological boundedness constraints vis-à-vis the EVH. This extends the property of 'boundedness', which is proposed to be iconic in the EVH (Wilbur 2008), to yet another syntactic object: the EVH is now shown to be a source of bounds 
to arguments, often realizing as +/- definite. This result supports the suggestion in Wilbur, Malaia, and Shay (2012) that the EVH should be generalized to a more general Visibility Hypothesis; their version was 'Sign languages express the boundaries of semantic scales by means of phonological mapping.' Here we see that such distinctions can be incorporated into functional items like quantifiers.

\section{References}

Abner, Natasha \& Ronnie B. Wilbur. 2017. Quantification in American Sign Language. In Denis Paperno \& Edward L. Keenan (eds.), Handbook of quantifiers in natural language: Volume II. 21-59. Berlin: Springer.

Davidson, Kathryn \& Deanna Gagne. 2014. Vertical representation of quantifier domains. Proceedings of Sinn und Bedeutung (SuB) 18. 110-127.

Kuhn, Jeremy, Carlo Geraci, Philippe Schlenker \& Brent Strickland. Under review. Boundaries in space and time: iconic biases across modalities. Manuscript, Institut Jean-Nicod.

Liskova, Elena Igorevna. 2017. Universal quantification in the nominal domain in American Sign Language. Austin, TX: University of Texas dissertation.

MacLaughlin, Dawn. 1997. The structure of determiner phrases: Evidence from American Sign Language. Boston, MA: Boston University dissertation.

Malaia, Evie \& Ronnie B. Wilbur. 2012. Kinematic signatures of telic and atelic events in ASL predicates. Language and Speech 55(3). 407-421. https://doi.org/10.1177/0023830911422201.

Malaia, Evie, Ronnie B. Wilbur \& Marina Milković. 2013. Kinematic parameters of signed verbs. Journal of Speech, Language and Hearing Research 56(5). 1677-1688. https://doi.org/10.1044/1092-4388(2013/12-0257).

Saral, Burcu \& Meltem Kelepir. 2019. Three signs, one common trait: 'All' a study on universal quantifiers in Turkish Sign Language. Paper presented at the $55^{\text {th }}$ Meeting of the Chicago Linguistic Society (CLS), Chicago, IL: The University of Chicago.

Saral, Burcu. 2019. The universal quantifier 'ALL' in Turkish Sign Language. Istanbul, Turkey: Boğaziçi University MA thesis.

Strickland, Brent, Carlo Geraci, Emmanuel Chemla, Philippe Schlenker, Meltem Kelepir \& Roland Pfau. 2015. Event representations constrain the structure of language: Sign language as a window into universally accessible linguistic biases. Proceedings of the National Academy of Sciences (PNAS) 112(19). 5968-5973. https://doi.org/10.1073/pnas.1423080112.

Wilbur, Ronnie B. 2008. Complex predicates involving events, time and aspect: Is this why sign languages look so similar? In Josep Quer (ed.), Signs of the time. Selected papers from TISLR 2004. 217-250. Hamburg: Signum Press.

Wilbur, Ronnie B., Evie Malaia \& Robin A. Shay. 2012. Degree modification and intensification in ASL adjectives. In Maria Aloni, Vadim Kimmelman, Floris Roelofsen, Galit Sassoon, Katrin Schulz \& Matthijs Westera (eds.), Logic, language and meaning. 92101. Berlin: Springer. 